\title{
Pratiques scolaires de l'écrit et temporalités dans le travail par modules
}
Academic literacy practices and temporalities of modular instruction use in the classroom
Prácticas escolares de la escritura y temporalidades en el trabajo por módulos

\section{Jean-Pierre Mercier}

Volume 45, numéro 3, 2019

Texte reçu le : 3 septembre 2018

Version finale reçue le : $1^{\mathrm{er}}$ octobre 2019

Accepté le : 23 décembre 2019

URI : https://id.erudit.org/iderudit/1069638ar

DOI : https://doi.org/10.7202/1069638ar

Aller au sommaire du numéro

\section{Éditeur(s)}

Revue des sciences de l'éducation

ISSN

1705-0065 (numérique)

Découvrir la revue

Citer cet article

Mercier, J.-P. (2019). Pratiques scolaires de l'écrit et temporalités dans le travail par modules. Revue des sciences de l'éducation, 45(3), 1-25.

https://doi.org/10.7202/1069638ar

\section{Résumé de l'article}

Cet article est issu d'une étude de cas ethnographique conduite auprès d'un groupe de jeunes mères de retour aux études en formation générale des adultes dans le cadre de la mesure Ma place au soleil. L'objectif de la présente étude était d'analyser les pratiques scolaires de l'écrit et les temporalités qui les accompagnent dans le travail par modules des cours de français, d'anglais et de mathématique que suivent ces femmes. L'enseignement modulaire individualisé est une formule reconnue comme centrale de ces cours en formation générale des adultes. Les résultats montrent la centralité de l'évaluation dans ces pratiques de l'écrit et leur maintien dans des temporalités du présent de la formation. L'entrainement à l'examen influence le maintien des pratiques de l'écrit dans le temps présent sans laisser voir la pertinence de nombreux buts autres que ceux liés à l'évaluation sanctionnée par l'État. Ces résultats, à défaut d'être généralisables, sont transférables aux contextes apparentés à celui enquêté. 


\section{Pratiques scolaires de l'écrit et temporalités dans le travail par modules ${ }^{1}$}

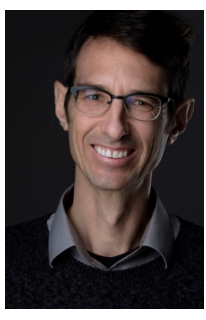

Jean-Pierre Mercier

Professeur

Université du Québec à Montréal

RÉSUMÉ-Cet article est issu d'une étude de cas ethnographique conduite auprès d'un groupe de jeunes mères de retour aux études en formation générale des adultes dans le cadre de la mesure Ma place au soleil. L'objectif de la présente étude était d'analyser les pratiques scolaires de l'écrit et les temporalités qui les accompagnent dans le travail par modules des cours de français, d'anglais et de mathématique que suivent ces femmes. L'enseignement modulaire individualisé est une formule reconnue comme centrale de ces cours en formation générale des adultes. Les résultats montrent la centralité de l'évaluation dans ces pratiques de l'écrit et leur maintien dans des temporalités du présent de la formation. L'entrainement à l'examen influence le maintien des pratiques de l'écrit dans le temps présent sans laisser voir la pertinence de nombreux buts autres que ceux liés à l'évaluation sanctionnée par l'État. Ces résultats, à défaut d'être généralisables, sont transférables aux contextes apparentés à celui enquêté.

MotS-CLÉS-pratiques de l'écrit, temporalités, enseignement modulaire, jeunes mères, ethnographie.

\section{Introduction}

Les pratiques de l'écrit, lire et écrire, s'étendent à presque toutes les pratiques scolaires contemporaines, qu'elles se concrétisent dans la scolarisation des jeunes ou dans celles des adultes. Le temps, celui qui a un caractère structurel dans l'organisation de nos vies comme celui qui renvoie à une certaine manière de vivre (Elias, 1996), est très souvent lié à ces pratiques de l'écrit et à bien d'autres : lire l'heure, le calendrier, écrire dans un agenda, prévoir la réalisation

\footnotetext{
${ }^{1}$ L'étude source d'où cet article est tiré a reçu le soutien financier et logistique du Centre d'études et de recherches sur les transitions et l'apprentissage. Les personnes et organismes qui ont participé à cette étude ou l'ont soutenue sont ici remerciés.
} 
d'un évènement, mais aussi écrire sur le passé, le présent ou le futur. Cette étude porte sur les pratiques scolaires de l'écrit et les temporalités, c'est-à-dire les cadres temporels d'organisation de l'existence (Boutinet, 2004), qui accompagnent ces pratiques dans l'enseignement modulaire individualisé. Elle s'intéresse spécifiquement aux pratiques de l'écrit et aux temporalités dans le travail par modules de jeunes mères de retour aux études à Ma place au soleil au moment de l'enquête de terrain.

Ma place au soleil est une mesure intersectorielle de soutien à l'insertion sociale et professionnelle des jeunes parents non diplômés désirant retourner aux études en formation générale des adultes. Le soutien offert par la mesure se concrétise en premier lieu à la formation générale des adultes. Plusieurs acteurs institutionnels et l'État québécois ont exprimé leurs préoccupations pour les jeunes mères non diplômées et leur formation. Celle-ci est d'ailleurs vue comme un moyen pour lutter contre la transmission intergénérationnelle de la pauvreté. Au moment de leur participation à cette mesure, ces jeunes femmes faisaient partie de la catégorie des jeunes adultes non diplômés.

Au Québec, de nombreux jeunes adultes non diplômés retournent aux études en formation générale des adultes. L'expression non diplômés est ici retenue pour désigner, parmi les jeunes adultes (entre 16 et 25 ans), ceux et celles qui ne détiennent pas de diplôme secondaire, général ou professionnel. Outre son utilité pour reconstituer et étudier un groupe vers lequel convergent plusieurs précarités (emploi, revenu, santé, littératie, logement, formation, notamment), cette expression est aussi moins normative et moins péjorative que l'expression peu scolarisés. Elle est aussi plus appropriée que le terme décrocheurs, non applicable à la situation de nombreux jeunes adultes ayant connu un parcours scolaire complexe ou en continuité de scolarisation de la formation générale des jeunes à formation générale des adultes.

Dans le système d'éducation québécois, la formation générale des adultes est un dispositif administratif et éducatif pour les adultes (Voyer, Potvin et Bourdon, 2014). Ce dispositif peut conduire à l'obtention du diplôme d'études secondaires. Ce diplôme et le diplôme d'études professionnelles, qui s'acquièrent au secteur de la formation professionnelle, sont les diplômes du secondaire correspondant à la «norme sociale de référence » (Gouvernement du Québec, 2002, p. 9) définissant la formation dite de base, selon la Politique gouvernementale d'éducation et de formation continue. Cette politique, qui date de 2002, est toujours en vigueur en 2019. 
En formation générale des adultes, l'apprentissage se fait souvent par l'intermédiaire de l'enseignement modulaire individualisé, mais plus souvent en formation de base diversifiée $\left(2^{\mathrm{e}}\right.$ cycle du secondaire) qu'en formation de base commune (alphabétisation, présecondaire et $1^{\text {er }}$ cycle du secondaire), selon nos observations. L'enseignement modulaire individualisé est une formule pédagogique qui combine enseignement modulaire et aide individualisée à la personne apprenante adulte. L'enseignement modulaire individualisé a pour caractéristique première d'être centré sur une répartition programmatique des contenus et des activités d'enseignement et d'apprentissage en unités indépendantes selon des modules dédiés à l'étude autonome (Goldschmid et Goldschmid, 1973). Or, cette formule pédagogique est traversée par des pratiques scolaires de l'écrit et par des temporalités qui accompagnent ces pratiques (Mercier, 2015). Ces pratiques et temporalités demeurent toutefois peu connues à ce jour, bien que l'enseignement modulaire individualisé soit présent au Québec en formation générale des adultes depuis le milieu des années 1970 (Baillargeon, Gascon et Rivard, 1992).

Connaitre les pratiques scolaires de l'écrit et les temporalités qui les accompagnent dans une formule comme l'enseignement modulaire individualisé aide à comprendre, en partie, comment le passé, le futur et le présent jouent dans la participation à la formation. Cette connaissance peut aussi orienter l'intervention dans les contextes de retour aux études de ces femmes ou, plus largement, dans les contextes de formation générale des adultes où l'enseignement modulaire individualisé est central. Cet article comble en partie le manque de connaissances sur ce sujet par la réponse qu'il apporte à la question suivante : quelles sont les pratiques scolaires de l'écrit et les temporalités de jeunes mères de retour aux études dans la mesure Ma place au soleil lorsque l'enseignement modulaire individualisé est la formule retenue?

\section{Contexte théorique : pratiques de l'écrit et temporalités}

L'enjeu épistémologique de cette étude consiste à penser que les pratiques de l'écrit jouent un rôle dans les temporalités vécues et que ces temporalités peuvent être analytiquement révélées par l'analyse des pratiques de l'écrit. Cette perspective épistémologique est inspirée des travaux de Bélisle (2012) sur l'écrit et l'apprentissage tout au long de la vie et de ceux de Boutinet (2004) sur les temporalités que l'analyse des pratiques sociales révèle. On conçoit donc ici que les 
pratiques de l'écrit et le temps se conjuguent dans les pratiques sociales et que le temps vécu s'exprime, au moins partiellement, par et dans les pratiques de l'écrit. On pense surtout ici à une certaine manière de vivre et d'expérimenter les temporalités (Hartog, 2012) dans les interactions faisant appel à l'écrit. C'est cette dimension du temps vécu dans les pratiques scolaires de l'écrit que l'article éclaire.

\subsection{Pratiques scolaires de l'écrit}

Par pratiques de l'écrit, on entend tout ce que les personnes font avec l'écrit. Il s'agit de pratiques sociales. Cette vision large inclut toute activité d'écriture ou de lecture, toutes les interactions faisant appel à l'écrit, toute utilisation complémentaire de l'oral et de l'écrit, de même que le contexte, toujours spécifique, influençant ce que les personnes font avec l'écrit (Bélisle, 2012 ; Papen, 2005). Les pratiques de l'écrit sont à la fois rendues possibles et contraintes par des éléments internes aux personnes (comme leurs initiatives, telles qu'elles les perçoivent, et leurs manières de penser et d'agir), ainsi que par des éléments qui leur sont externes (comme les demandes externes et le contexte) (Bélisle, 2007).

Les pratiques scolaires de l'écrit sont des pratiques dominantes de l'écrit (Barton et Hamilton, 1998), orientées vers les buts formels de l'institution plus que vers ceux des personnes ou des groupes. Ces pratiques bénéficient d'une plus grande légitimité sociale que les pratiques de l'écrit de la vie courante, car elles sont normées et codées par l'institution scolaire dans les politiques, règles, programmes, manuels, etc. Dans les pratiques scolaires de l'écrit, les connaissances sont divisées, objectivées et évaluées selon les disciplines ou spécialités d'enseignement. Les connaissances sont exposées et apprises explicitement, de façon hiérarchique et séparément des contextes de la vie courante (Barton et Hamilton, 1998). Ces pratiques scolaires de l'écrit comportent des caractéristiques de la forme scolaire qui instaure un rapport distancié au savoir dans un lieu et un temps spécifiques, notamment par le recours à l'écrit (Vincent, Lahire et Thin, 1994).

Le terme écrit désigne ici tout système langagier de code graphique sur support (Bélisle et Bourdon, 2006). L'écrit est aussi considéré comme un outil, au sens vygotskien du terme (Vygotski, 1997), approprié à un but dans l'activité des personnes. Comme outil, il peut jouer un rôle dans le processus de rationalisation et d'objectivation du temps vécu, que ce soit dans 
l'organisation, la mise en ordre et la coordination des évènements ou dans le futur et dans le passé qui entrent en résonance avec le présent vécu par les personnes (Goody, 1979).

\subsection{Temps et temporalités}

Le temps est une invention humaine. Les manières de le concevoir changent historiquement et culturellement, comme l'a montré Elias (1996). Einstein (1905/1923) a aussi souligné le caractère fondamentalement humain du temps. Pour ce physicien, traduit ici librement à partir de son article en anglais d'abord publié en allemand, la description mathématique du temps «n'a aucun sens physique » (p. 2) indépendant du sens que nous lui attribuons. Comme il le souligne, le temps se rapporte toujours à nos «jugements d'évènements simultanés» (p. 2). Inventé, changeant, résultat de nos jugements plutôt que d'une donnée objective existant indépendamment de ceux-ci, le temps est appris et expérimenté tout au long de la vie et dans toutes ses dimensions (Bourdon et Bélisle, 2005).

La notion de temporalités, comme la définit Boutinet, permet de rendre compte des temps associés aux pratiques scolaires de l'écrit. Pour Boutinet, les temporalités sont des «cadres temporels» $(2004$, p. 5) que les personnes se donnent ou qui leur sont imposés par leur environnement pour organiser leur existence quotidienne. On se réfère ici à un modèle polychronique des temporalités plutôt qu'à un modèle monochronique et linéaire qui engage une sorte d'irréversibilité du temps. Le modèle polychronique permet de révéler le temps vécu par les jeunes mères, tout en prenant en compte le temps institutionnel, tels que l'un et l'autre se manifestent dans les données produites dans le temps présent. Ce modèle prend différentes formes: "celle des choses passées présentement remémorées, celle des choses futures présentement anticipées, celle des choses momentanées présentement expérimentées » (Boutinet, 2004, p. 44).

\subsection{Pratiques de l'écrit et temps}

Le système d'éducation de la société québécoise se distingue en partie de celui des autres sociétés avec lesquelles elle partage des traits culturels (Bourdon et Bélisle, 2015). Pour cette raison, la recension des textes scientifiques s'est centrée sur les recherches conduites au Québec auprès d'une proportion significative de jeunes adultes non diplômés participant à la formation, soit en 
contexte d'éducation non formelle, comme dans des organismes communautaires, soit en formation générale des adultes, un contexte d'éducation formelle. Considérant le petit nombre de textes scientifiques qui fournissent des éléments de réponse à la question de la présente étude, il faut rappeler ici l'importance d'investir en recherche sur les pratiques ou approches d'enseignement et d'apprentissage de la lecture et de l'écriture (mais aussi de la lecture et de l'écriture pour apprendre) en éducation des adultes au Québec, notamment pour les adultes en situation de précarité dont font partie les non-diplômés du secondaire. Comme le signalent les auteures d'une recension récente (Ouellet, Dubeau, Dubé et Voyer, 2017), le manque de connaissances sur ce sujet est important.

Certaines des recherches recensées ne portent pas spécifiquement sur les pratiques de l'écrit, mais évoquent le temps dans l'enseignement modulaire individualisé : soit les perceptions de formatrices jugeant que cette formule respecte le rythme d'apprentissage (Bourdon et Bélisle, 2005), soit les perceptions des jeunes adultes qui, pour la même raison, apprécient la formule (D’Ortun, 2009 ; Rousseau, Théberge, Bergevin, Tétreault, Samson, Dumont et Myre-Bisaillon, 2010).

Mais l'enseignement modulaire individualisé peut aussi être démotivant quand les classes comptant beaucoup d'élèves empêchent d'avancer à son rythme (Potvin et Leclercq, 2014).

On repère cependant un corpus d'études et de recherches empiriques dont les résultats aident à comprendre comment des pratiques de l'écrit et des temps divers s'articulent dans la participation à la formation des jeunes adultes non diplômés. Pour celles et ceux participant à la formation dans des organismes communautaires d'employabilité ou d'alphabétisation, écrire et lire, ainsi que les interactions autour de l'écrit, les amènent à revenir sur leurs expériences ou leurs acquis. L'écrit peut jouer un rôle important dans le travail de mémoire de ces jeunes adultes (Bélisle, 2003). Il leur permet de prendre du recul face à leurs expériences et aide à tirer des apprentissages susceptibles d'être mobilisés dans les situations actuellement vécues ou anticipées, voire à prendre confiance en leur potentiel (Bélisle, 2004; Desmarais, 2003 ; Desmarais et Lamoureux, 2012 ; Thériault, 2015).

En contexte de formation générale des adultes, les travaux de Bélisle et Rioux (Bélisle et Rioux, 2016 ; Rioux et Bélisle, 2012) montrent le rôle de l'écrit dans la démarche des univers de compétences génériques, un dispositif de reconnaissance des acquis et des compétences de la 
formation générale des adultes permettant d'obtenir des unités pour l'obtention du diplôme d'études secondaires. L'écrit amène les adultes, jeunes comme moins jeunes, à puiser dans leur passé et à repérer parmi leurs expériences et acquis ceux qu'ils ou elles veulent faire valoir, tout comme il les aide à anticiper les besoins de formation. L'étude source (Mercier, 2015) d'où est tirée la présente étude a permis de documenter une variété de pratiques de l'écrit et de temporalités chez les jeunes mères de retour en formation à Ma place au soleil. Elle a permis de montrer que des organismes non scolaires intervenant dans le cours d'intégration socioprofessionnelle contribuent à certaines pratiques de l'écrit de la vie courante auxquelles ces femmes attachent une temporalité d'anticipation du travail domestique (soin, entretien) (Mercier et Longo, 2017). Cette étude source est aussi la seule, sauf erreur, à avoir documenté des pratiques de l'écrit et des temporalités dans les cours de formation générale des adultes où l'enseignement modulaire individualisé est présent. L'objectif de la présente étude est d'analyser les pratiques scolaires de l'écrit et les temporalités qui leur sont attachées dans l'enseignement modulaire individualisé. Cette étude ne revient cependant pas sur le phénomène d'imbrication des temps et des pratiques de l'écrit scolaires et domestiques quand cette formule est centrale, car il a été analysé ailleurs (Mercier et Longo, 2017).

\section{Méthodologie}

L'étude source a été approuvée par le Comité d'éthique de la recherche en éducation et sciences sociales de l'Université de Sherbrooke. La stratégie méthodologique est l'étude de cas ethnographique (Merriam, 1998). Cette stratégie concorde, d'une part, avec la problématique et la question de recherche soulevée. Elle permet effectivement de partir du cas initialement établi (Mercier, 2015), celui des pratiques de l'écrit de jeunes mères de retour en formation à Ma place au soleil, pour dégager spécifiquement les pratiques scolaires de l'écrit et les temporalités qui les accompagnent dans l'enseignement modulaire individualisé. D'autre part, l'ethnographie et ses méthodes sont appropriées pour l'étude des pratiques de l'écrit. Elles permettent de produire et d'analyser des données sur ce que les personnes font avec l'écrit et de mettre ces usages en relation avec les éléments du contexte qui influencent leurs pratiques de l'écrit (Papen, 2005). L'enquête de terrain a été conduite dans un centre d'éducation des adultes francophone, en milieu urbain, où les jeunes mères participaient à la formation en groupe $(n=31)$. L'enseignement 
modulaire individualisé était la formule centrale des cours de français, d'anglais et de mathématique. Ces femmes participaient également à un cours d'informatique et à un cours d'intégration socioprofessionnelle. Un article est par ailleurs programmé sur les pratiques de l'écrit et temporalités liées au choix d'un métier dans ce dernier cours.

Les sources d'information (S) ont été produites par observation participante (S1), photographie des lieux (S2), entretiens informels (S3), collecte de documents par choix raisonné parmi ceux utilisés par les jeunes mères ou présents dans l'interaction (S4) et des documents institutionnels (S5), entretien semi-dirigé avec treize jeunes mères sélectionnées en fonction de la représentativité de leur niveau de scolarité dans le groupe au moment de conduire cet entretien (S6) et entretien semi-dirigé avec cinq personnes intervenantes (S7), puis rencontres de restitution avec de jeunes mères (S8) et avec les personnes intervenantes (S9).

Les deux guides d'entretien semi-dirigé comportaient des sections pour traiter spécifiquement les pratiques scolaires de l'écrit, l'évaluation et l'enseignement modulaire individualisé dans les cours de français, d'anglais et de mathématique. Les analyses préliminaires présentées lors des deux rencontres de restitution ont porté sur ces thèmes étant donné leur prédominance dans le corpus de données. Le guide des rencontres de restitution a été préparé pour traiter ces thèmes.

L'ensemble de ces méthodes ont été déployées pendant l'enquête de terrain, du début octobre 2012 à la fin mai 2013, au cours de 49 séances de terrain de 3 h 30 en moyenne. Ces séances ont été réparties en six vagues d'enquête. Le tableau 1 représente le déroulement de l'enquête selon les méthodes déployées par vague. 
Tableau 1

Méthode de production des données déployées par vague de l'enquête de terrain

\begin{tabular}{|c|c|c|c|c|c|c|}
\hline \multirow[t]{2}{*}{ Méthodes } & \multicolumn{6}{|c|}{ Vagues (V) de l'enquête de terrain (2013-2013) } \\
\hline & $\begin{array}{c}\mathrm{V} 1 \\
9 \text { séances } \\
\text { oct. }\end{array}$ & $\begin{array}{c}\mathrm{V} 2 \\
10 \text { séances } \\
\text { nov.-déc. }\end{array}$ & $\begin{array}{c}\mathrm{V} 3 \\
14 \text { séances } \\
\text { jan. }\end{array}$ & $\begin{array}{c}\mathrm{V} 4 \\
6 \text { séances } \\
\text { fév. }\end{array}$ & $\begin{array}{c}\mathrm{V} 5 \\
6 \text { séances } \\
\text { mars-avr. }\end{array}$ & $\begin{array}{c}\mathrm{V} 6 \\
4 \text { séances } \\
\text { mai }\end{array}$ \\
\hline Observation & $\mathrm{x}$ & $\mathrm{X}$ & $\mathrm{x}$ & $\mathrm{x}$ & $\mathrm{x}$ & $\mathrm{x}$ \\
\hline Photographie & $\mathrm{x}$ & $\mathrm{x}$ & $\mathrm{x}$ & $\mathrm{x}$ & $\mathrm{x}$ & $\mathrm{x}$ \\
\hline Entretiens informels & $\mathrm{x}$ & $\mathrm{x}$ & $\mathrm{x}$ & $\mathrm{x}$ & $\mathrm{x}$ & $\mathrm{x}$ \\
\hline Collecte de documents & $\mathrm{x}$ & $\mathrm{x}$ & $\mathrm{x}$ & $\mathrm{x}$ & $\mathrm{x}$ & $\mathrm{x}$ \\
\hline Entretiens semi-dirigés & & & $\mathrm{x}$ & & & \\
\hline Restitution euristique & & & & & & $\mathrm{x}$ \\
\hline
\end{tabular}

Les sources d'information ont été téléchargées dans le logiciel NVivo qui a servi d'outil d'aide à l'analyse. Les données analysées pour la présente étude sont celles liées aux pratiques scolaires de l'écrit dans l'enseignement modulaire individualisé. Il s'agit de données synchroniques correspondant approximativement à l'année scolaire pendant laquelle les jeunes mères retournent aux études. Ces données ont été l'objet d'une analyse thématique suivant une « logique inductive et délibératoire » (Savoie-Zajc, 2004, p. 140). Suivant cette logique, le cadre théorique a guidé l'analyse dès le début de la production des sources jusqu'à l'introduction des résultats de l'étude. Cette logique a aussi permis de demeurer ouvert à l'ajout de thèmes émergents là où les données révélaient les dimensions inédites des pratiques étudiées. Cette stratégie d'analyse a permis de révéler une temporalité générale qui accompagne les pratiques scolaires de l'écrit dans le travail par modules. Les données et leur analyse ont permis de reconstituer les pratiques scolaires de l'écrit et temporalités exposées ici.

\section{Pratiques scolaires de l'écrit et temporalités dans le travail par modules}

La catégorie des pratiques scolaires de l'écrit distingue analytiquement deux sous-catégories, indissociables dans les pratiques effectives. Elle regroupe les pratiques de l'écrit pour la réalisation des modules et celles pour l'évaluation. Des temporalités spécifiques sont attachées à cet ensemble de pratiques. 


\subsection{Pratiques de l'écrit pour le travail par modules}

Les données montrent une relative adhésion des jeunes mères à ces pratiques. Comme Élise (18 ans, sec. 3) l'évoque abruptement: Je vais en faire un peu [d'exercices dans mon cahier], mais je ferai pas toute (S1, Élise, 2012-10-18).

Les cahiers correspondent à la principale condition matérielle des modules. L'écrit s'y présente sous diverses formes de textes : énoncés, consignes, définitions, mises en situation, schémas, graphiques, cartes géographiques, réponses et corrigés que les apprenantes écrivent ou lisent, accompagnés d'autres traces de l'activité d'écriture ou de lecture, comme les soulignements, les annotations. On y trouve aussi des textes ou sections de textes plus longs: articles et récits originaux, extraits de périodiques, de livres, de sites internet et ceux que les apprenantes écrivent. Les textes d'un module se trouvent parfois sur d'autres supports que les cahiers : feuilles brochées ou didacticiel, fichiers de textes oralisés ou vidéos documentaires.

Les apprenantes peuvent faire les exercices de leur cahier à leur rythme à la suite l'un de l'autre ou dans un ordre qui leur convient mieux, soit parce qu'elles les trouvent intéressants ou les comprennent ou, au contraire, parce qu'elles ne les comprennent pas et préfèrent attendre une explication individualisée avant de les continuer. L'aide individualisée de la personne enseignante est centrale dans ces pratiques. On observe souvent la personne enseignante lire à haute voix à côté de l'apprenante, puis donner une explication. L'explication est souvent accompagnée d'un moment où la personne enseignante recourt à l'écrit en montrant comment faire l'exercice à l'apprenante ou en faisant une partie avec elle. Comme le dit Julie (22 ans, sec. 2) en ce qui concerne l'aide qu'elle demande à l'enseignante de français : je lui dis, là madame, aidez-moi, pis faites-moi en deux trois [des exercices] avec moi. (Rire) Fait que là, elle en fait deux trois là, avec moi, là ça m'aide (S6, Julie). Ces moments ressemblent à une « modélisation » (Legendre, 2005, p. 907) de l'écriture ou de la lecture aidant à apprendre le contenu en reproduisant un modèle.

En même temps, dans les pratiques de l'écrit pour le travail par modules, la personne enseignante n'est pas toujours disponible. Les observations montrent que les apprenantes peuvent attendre cette aide pendant de longues minutes à leur place ou au bureau de la personne enseignante avant qu'elle se libère, occupée qu'elle est à aider une autre apprenante. Mais assez souvent, les apprenantes n'attendent pas et demandent de l'aide à leurs pairs du même niveau de 
scolarité ou d'un niveau plus élevé. Cette tendance forte dans les données d'observation se confirme dans le propos des apprenantes, lesquelles ont une bonne connaissance du niveau de scolarité de leurs pairs, des cahiers qu'elles ont faits et de ceux qu'elles sont en train de faire, ce qui leur permet de se situer par rapport aux autres et contribuerait à une certaine " émulation", comme l'ont observé Bourdon et Bélisle (2011, p. 17) dans un contexte apparenté.

\subsection{Pratiques de l'écrit pour l'évaluation}

Les pratiques de l'écrit pour l'évaluation sont au cœur du travail par modules. Elles ont pour but de participer à l'évaluation en tant qu'apprentissage (assessment as learning) sanctionné (Torrance, Colley, Garratt, Jarvis, Piper et Ecclestone, 2005). Les données montrent une forte adhésion des apprenantes à ces pratiques : les examens on prend ça plus à cœur que / que quand on travaille dans nos livres parce qu'on le sait que [...] c'est ça qui compte (S6, Alexia).

Les prétests sont des outils qui soutiennent l'apprentissage en vue de l'examen. Ils permettent de vérifier si les apprentissages visés et sanctionnés sont atteints : ça t'aide, dans le fond, à avoir de bonnes notes dans tes examens (S6, Amélie). Dans les prétests, l'écrit se présente sous forme d'exercices apparentés à ceux des cahiers : mises en situation, énoncés, consignes, questions, schémas, tableaux et espaces pour écrire les réponses, qui sont séparés, numérotés et souvent accompagnés de points attribués par question. L'étude source n'a pas prévu recueillir d'examens. Toutefois, les données permettent d'avancer qu'ils s'apparentent aux prétests. Parlant d'un prétest, une personne enseignante dit que l'examen est sensiblement pareil (S7). Une autre parle d'un prétest comme d'un faux examen (S7).

Dans les pratiques de l'écrit pour l'évaluation, l'écrit est un outil qui soutient l'entrainement à l'examen (exam coaching), lequel contribue fortement à fondre l'apprentissage dans l'évaluation. De telles pratiques de l'écrit ont également été observées par Ivanic, Edwards, Barton, Martin-Jones, Fowler, Hugues et Mannion (2009), mais dans des contextes de formation professionnelle (vocational education) au Royaume-Uni. Torrance et ses collègues (2005) définissent l'entrainement à l'examen comme tout soutien donné à travers le morcèlement, l'interprétation et l'élucidation des critères ou des aspects de l'évaluation de même que

l'intervention dans l'évaluation permettant aux personnes apprenantes de comprendre le processus d'évaluation. Dominant à la fois l'enseignement et l'apprentissage, l'entrainement à 
l'examen contribue au phénomène de l'évaluation en tant qu'apprentissage, au sens où il permet d'apprendre l'évaluation en répétant les exercices qui lui correspondent.

Le plus souvent, chaque module se répète suivant la même séquence d'activités d'écriture et de lecture rapidement orientées vers l'évaluation : faire des exercices du cahier, un ou plusieurs prétests en vue de faire l'examen. Cette séquence fonctionne comme une boucle parfaite dont la fin, dans les deux sens du terme, est inscrite dans l'évaluation annoncée dès le début de chaque module : faire l'examen. Quand une apprenante commence le cahier, des indications lui sont données quant aux examens à faire, comme le nombre et les types d'examens, les objets évalués, le niveau de difficulté à anticiper et les attentes en matière d'écriture ou de lecture pour s'y préparer : je commence toujours avec ce qu'elles vont avoir à faire à la fin. Je leur explique un peu ce qu'on leur demande finalement à l'examen en leur soulignant l'importance, justement, des activités qui vont les préparer à tout ça (S7, personne enseignante). On voit aussi, dans des couvertures ou des pages des cahiers, des numéros de pages écrits, encerclés ou barrés indiquant les exercices ou parties d'exercices que la personne enseignante juge importants de faire ou de ne pas faire pour la préparation à l'examen. Ces exercices sont choisis par la personne enseignante parce qu'ils correspondent à une faiblesse de l'apprenante ou à un objet d'évaluation du module. On observe aussi que des apprenantes (par exemple, Élodie, 25 ans, sec. 3 ; Gaëlle, 21 ans, sec. 4 ; Stéphanie, 21 ans, sec. 2) prennent des initiatives en demandant des exercices sur un objet précis qu'elles veulent bien comprendre.

Lors de la correction des exercices du cahier ou du prétest par la personne enseignante, l'écrit donne des indications et laisse des traces relativement aux attentes pour l'examen. Le propos des personnes enseignantes montre les traces de ces indications dans la correction des exercices de la même façon (S7) qu'à l'examen, comme si c'était un examen (S3), décortiquer un texte [et] toutes les questions pertinentes pour l'examen (S7). Il en va de même pour les prétests : je le fais [corriger le prétest] tout le temps avec [nomme la personne enseignante] pour être sure de ne pas me donner des points que j'ai pas à avoir (S6, Éliane). Ces interactions faisant appel à l'écrit donnent lieu à l'explication et à l'élucidation des justifications de la correction et des attentes de l'examen de sorte que l'apprenante les comprenne.

Ces indications dans l'entrainement à l'examen sont aussi présentes dans les corrigés. Dans ceux-ci, l'écrit permet de voir, littéralement, les réponses attendues : voir l'explication écrite 
dans mon cahier, je vais pouvoir la comprendre (S6, Ariane); pour voir à quoi ressemblent les réponses (S3, Éliane); voir si j’ai la bonne réponse (S6, Alexia); voir si c'est correct (S6, Julie).

[Dans le corrigé] je vais lire ce qui est écrit, pis des fois ils vont me montrer comment faire, mettons la démarche pis tout ça pour arriver à faire euh, mettons un calcul. Fait que je vais le lire, pis après ça ils vont donner comme des situations pour les refaire, pis là ben, je vais les faire pis des fois ben, je vais retourner voir c'est comment qu'ils disaient pour procéder pour le faire (S6, Éloïse)

Les propositions faites par les personnes enseignantes concernant l'utilisation du corrigé peuvent aller dans le même sens, soit proposer de se servir du corrigé juste pour se corriger ou [...] pour s'aider à comprendre (S7) ce qu'il faut faire.

Un prétest terminé peut aussi aider à faire un prétest additionnel : tous les prétests que [nomme la personne enseignante] m'a fait faire, ben je vais les garder, comme ça, quand je bloque [...] ben je peux aller relire, pis dire, ah c'est vrai, c'est de même que je faisais (S6, Éloïse). Les apprenantes semblent faire un ou deux prétests avant d'aller à l'examen. Certaines (Amélie, 21 ans, sec. 4 ; Gaëlle, 21 ans, sec. 4 ; Noémie, 24 ans, présec. ; Sandra, 21 ans, sec. 5) parlent, selon la matière, de trois ou quatre prétests. Si, après les prétests, elle échoue à l'examen, l'apprenante peut faire de nouveau un ou plusieurs prétests pour se préparer à la reprise, comme le disent certaines (Amélia, 19 ans, présec.; Ariane, 25 ans, sec. 4 ; Éloïse, 19 ans, sec. 1), ou faire des exercices ciblés. L'écrit se répétant à travers les exercices fait en sorte que les apprenantes apprennent le contenu du module par la répétition et la reproduction des attentes portant sur l'examen.

Ici, comme l'a montré Goody (1979), la matérialité de l'écrit peut jouer un rôle important, sur le plan cognitif, dans l'apprentissage. Par la trace qu'il laisse, l'écrit offre cette possibilité de reproductibilité. Tracées à l'écrit dans les corrections ou les corrigés, les attentes deviennent visibles. L'écrit aide ainsi les apprenantes à répondre à ces attentes en suivant leurs traces, en les répétant et en les reproduisant tout au long de la séquence exercices-prétests-examen.

En même temps, dans cette séquence d'entrainement à l'examen, certaines activités d'écriture ou de lecture sont perçues par les apprenantes comme plus pertinentes que d'autres. Pour Gaëlle (21 ans, sec. 4), les prétests la préparent à l'examen, plus adéquatement que ne le font les exercices de son cahier. Comme elle le dit, c'est en faisant ses prétests qu'elle a pu 
découvrir vraiment ce qui [la] boguait pour l'examen, avant d'ajouter qu'ils lui ont permis de pratiquer juste ce [avec quoi elle a] de la misère au lieu de tout pratiquer [...]. Ce [qu'elle sait] déjà, continuer à pratiquer, ça sert pas. Annabelle (19 ans, sec. 5) et Élise (18 ans, sec. 3) disent la même chose. Pour elles, ce sont les prétests qui les aident à se préparer à l'examen, alors qu'elles jugent que les exercices de leur cahier ne sont pas utiles pour cette préparation : Tu sais, dans tes cahiers, [...] c'est vraiment détaillé. Ils te demandent des questions sur tout, tout, tout, là. Dans ton examen là, tu as juste, euh, les questions qui valent la peine d'être répondues là (S8, Anabelle); mon cahier là que je fais, il ne me prépare même pas pour l'examen (S8, Élise).

Dès lors, l'entrainement à l'examen donne lieu à de nombreuses négociations entre l'apprenante et la personne enseignante, orientant l'activité d'écriture ou de lecture vers les contenus jugés nécessaires d'être appris pour l'examen.

[Au bureau de l'enseignante, assise à côté d'elle, Élise dit:] Si je fais l'examen, je vais comprendre ce que je comprends pas. Là, je fais ce que je comprends. » Anne répond: "Moi je peux pas t'envoyer à l'examen comme ça. Je t'enverrai pas à l'examen en début d'année. (S1, 2012-1018)

Questionnées sur ces négociations - qui se balancent entre un futur proche souhaité par les apprenantes (aller faire l'examen) et les exigences institutionnelles du présent (faire les apprentissages supposés non maitrisés qui y préparent) - des apprenantes parlent de ce qu'elles perçoivent devoir faire et de ce qu'elles perçoivent ne pas être obligées de faire dans le cahier : demander ou s'entendre sur ce qui n'était pas vraiment à l'examen [...] des choses qui étaient plus ou moins essentielles (S6, Alexia), ce qui est obligatoire vu que c'est dans l'examen (S6, Amélia), enlever ce qui est jugé inutile [vu] qu'ils ne le demandent pas à l'examen (S6, Ariane). Il se dégage du propos de ces apprenantes une vision utilitaire de la formation, au sens où elle est jugée utile pour l'obtention du diplôme d'études secondaires ou des préalables à la formation professionnelle ou technique, possiblement sans que les contenus d'apprentissage soient jugés utiles au-delà de la formation et du domaine scolaire. Cette vision peut être nourrie en partie par l'intervention des personnes enseignantes. L'une peut cibler la matière nécessaire (S7), l'autre peut pointer les pages dans le cahier avec les choses essentielles (S7) ou une autre préparer un document avec le contenu pouvant faire partie des exigences qu'ils vont leur demander à l'examen (S7). 


\subsection{Temporalités du présent}

Les temporalités du présent correspondent au cadre temporel général des pratiques scolaires de l'écrit dans le travail par modules. C'est le cadre temporel que les apprenantes se donnent, ainsi que celui qui leur est institutionnellement imposé. Ces temporalités se révèlent analytiquement sur plusieurs facettes de ces pratiques. C'est le temps présent qui prédomine dans le propos d'Ariane et d'Élise. On est là pour travailler dans nos cahiers (S6, Ariane). Les trois premiers mots d'Ariane campent ces pratiques de l'écrit dans le moment présent de la réalisation des modules. Lire et écrire sert à faire les modules sans anticipation des buts et des contenus d'apprentissage qu'ils comportent. Dès lors, il n'y a pas de raison de revenir sur ces buts et ces contenus, sauf pour ceux qui concernent l'entrainement à l'examen. Élise évoque certes le futur lorsqu'elle dit: Je vais en faire un peu. Il s'agit toutefois d'un futur proche qui pose d'emblée une limite temporelle à cette pratique : mais je ferai pas toute (S1, Élise, 2012-10-18). Le jeu de négociations s'enclenche ainsi sur les exercices à faire et ceux qu'il n'est pas nécessaire de faire pour répondre aux attentes avant de faire l'examen. Les pratiques de l'écrit dans la réalisation des modules balisent les exercices à faire. Conséquemment, elles permettent d'estimer le temps qu'il faudra pour les terminer.

Une autre temporalité du présent s'exprime dans l'attente d'une explication, sorte de temps qui suspend momentanément la réalisation des exercices. Lorsque les apprenantes n'attendent pas et obtiennent immédiatement une explication, le présent demeure le cadre temporel de réalisation du module du fait, justement, de l'immédiateté de la réponse. Ainsi, les pratiques de l'écrit pour le travail par modules s'adaptent plus ou moins au rythme d'apprentissage des jeunes mères, ce qui correspond aussi à leur préférence pour l'enseignement modulaire individualisé, repérable dans le propos de plusieurs apprenantes (Alexia, 21 ans, sec. 4 ; Ariane, 25 ans, sec. 4 ; Éliane, 21 ans, sec. 4 ; Stéphanie, 21 ans, sec. 2).

Avancer à son rythme semble vouloir dire avancer sans se sentir ralentie ni poussée par le groupe. En groupe il faut que tu attendes après les autres (S6, Alexia) ou ça va trop vite (S6, Stéphanie), alors que dans une formule individualisée il n'y a personne qui te stresse (S6, Alexia) ou tu peux aller plus vite (S6, Stéphanie). Quant aux personnes enseignantes, leurs interventions s'adaptent le plus souvent au rythme d'apprentissage des jeunes mères. Les interventions 
rejoignent ainsi les pratiques andragogiques promues par les programmes de formation et les documents d'encadrement de la mesure Ma place au soleil auxquels l'enquête de terrain a permis d'accéder, de même qu'elles entrent globalement en cohérence avec le « cadre souple » (Conseil du statut de la femme, 2004, p. 73) pensé pour la conciliation études-famille des jeunes parents participant à la mesure. Les données empiriques de l'étude source ont d'ailleurs permis de montrer comment s'actualise, en partie, la conciliation études-famille chez les jeunes mères rencontrées, notamment lorsque l'enseignement modulaire individualisé est la formule centrale (Mercier et Longo, 2017).

Certes, l'écrit ne permet pas à lui seul le respect du rythme individuel d'apprentissage. C'est l'enseignement modulaire individualisé qui joue un premier rôle sur ce plan. Cette formule réduit significativement l'obligation de synchronisation de l'activité individuelle à celle du groupe. Chaque apprenante fait son module à son rythme, sans suivre celui du groupe. Bien que les apprenantes se retrouvent, suivant l'horaire des cours de français, d'anglais et de mathématique, dans le même lieu et dans le même temps pour faire les modules - sans mentionner les occasions évoquées du travail par modules réalisé à la maison ou ailleurs - l'écrit, comme outil dans l'enseignement modulaire individualisé, joue aussi un rôle central dans l'adaptation au rythme individuel. Il permet la désynchronisation de l'activité d'écriture et de lecture de chaque individu (chaque apprenante écrit et lit individuellement, en partie du moins, dans son cahier) par rapport à celle du groupe (toutes les apprenantes du groupe écrivent et lisent dans leur cahier à la même case horaire) et, conséquemment, contribue au respect du rythme individuel.

L'autre temporalité du présent se trouve dans la séquence répétitive de réalisation de chaque module. Les apprenantes font les modules l'un à la suite de l'autre et les enchainent suivant les mêmes pratiques de l'écrit qui se répètent suivant la même séquence : faire une partie des exercices, quelques prétests, puis l'examen. Cette temporalité s'apparente à un temps archaïque, au sens où l'a proposé Éliade (1966) et comme l'ont aussi noté Bourdon et Bélisle (2005) dans un contexte apparenté. Dans ce temps qui se répète vit l'espoir que les exercices soient reproduits lors de l'examen. C'est la répétition des exercices qui confère l'espoir de savoir comment les refaire à l'examen. Lorsque celui-ci est réussi, la même séquence se répète en commençant le module suivant, suivant la même séquence enchainée dans le présent jusqu'à la 
réalisation de tous les modules requis pour libérer l'avenir meilleur, promis par l'obtention du diplôme ou l'accès à la formation professionnelle.

\section{Discussion}

Les résultats traitant des pratiques de l'écrit et du temps dans les recherches recensées montrent que certaines de ces pratiques amènent les jeunes adultes non diplômés à mobiliser le passé, le présent et le futur, notamment dans des contextes éducatifs non formels en milieu communautaire. On pourrait penser que c'est là un trait distinctif entre les pratiques de l'écrit en contextes d'éducation non formelle (Bélisle, 2004 ; Desmarais, 2003 ; Desmarais et Lamoureux, 2012 ; Thériault, 2015) et les pratiques scolaires de l'écrit et les temporalités en formation générale des adultes, où l'on rencontre de nombreux jeunes adultes non diplômés. Mais des recherches montrent que certaines pratiques de l'écrit en formation générale des adultes amènent les adultes non diplômés, jeunes comme moins jeunes, à mobiliser ces trois catégories générales du temps : le passé, le présent et le futur. C'est le cas, notamment, des pratiques de l'écrit et des temps à l'œuvre dans les univers de compétences génériques (Bélisle et Rioux, 2016; Rioux et Bélisle, 2012) et dans un cours comme celui d'intégration socioprofessionnelle (Mercier et Longo, 2017).

Comparativement aux résultats de ces recherches, ceux de la présente étude montrent la prépondérance de l'évaluation dans les pratiques scolaires de l'écrit pour le travail par modules. Ce phénomène n'avait pas été documenté par les études ou recherches recensées conduites en contexte de scolarisation des jeunes adultes en formation générale des adultes. La quasiexclusivité accordée à l'évaluation, et spécifiquement à celle sanctionnée par l'État pour l'obtention du diplôme d'études secondaires ou des préalables à la formation professionnelle ou technique, joue un rôle central dans le maintien des pratiques de l'écrit de réalisation des modules dans le temps présent. Une telle évaluation et les pratiques de l'écrit qui l'accompagnent sont absentes des contextes d'éducation non formelle mentionnés. Mais l'évaluation et les pratiques de l'écrit sont bien présentes dans le dispositif des univers de compétences génériques, lequel conduit à l'obtention d'unités comptables pour le diplôme d'études secondaires. La temporalité du présent n'est pourtant pas celle qui domine dans ce contexte. L'une des différences majeures entre les pratiques de l'écrit dans ce dispositif et les pratiques scolaires de l'écrit dans 
l'enseignement modulaire individualisé réside dans l'entrainement à l'examen, suivant la séquence exercices-prétest-examens, déclenchée en commençant chaque module. La répétition de ces pratiques de l'écrit, inhérente à l'entrainement, fonctionne comme une boucle qui restreint les possibilités de penser et de vivre le passé comme le futur au-delà du moment présent.

Mais on aurait tort de chercher les influences de cette temporalité, qui maintient les pratiques de l'écrit pour le travail par modules dans le présent, uniquement dans les attitudes et initiatives des apprenantes ou encore dans les demandes des personnes enseignantes. Des influences externes à plus grande échelle jouent un rôle significatif sur ces pratiques et ces temporalités.

Du côté des programmes, bien qu'ils visent à mettre en œuvre des «situations de vie authentiques» (Ministère de l'Éducation, du Loisir et du Sport, 2007, p. 15) rejoignant les situations de la vie courante que les adultes sont réputés rencontrer, ces situations rejoignent assez peu les préoccupations des jeunes mères ayant participé à cette étude. Les données ne fournissent aucun indice laissant penser que ces femmes transfèrent les textes ou leurs usages de l'écrit dans les modules jusque dans la vie courante ni que les situations d'apprentissage des modules, thématiquement liées aux préoccupations effectives des jeunes mères, rejoignent effectivement leurs préoccupations au-delà du temps court de la réalisation des modules. $\mathrm{Au}$ contraire, questionnées sur les situations d'apprentissage des modules qu'elles sont en train de faire et leur ressemblance avec les situations de la vie courante qu'elles rencontrent effectivement (budget du ménage, déménagement, achats à l'épicerie, par exemple), les jeunes mères répondent qu'il n'y a pas de ressemblance. Dans les situations d'apprentissage du module, il faut que tu résoudes des problèmes (S6, Amélia), c'est une affaire qu'il y avait dans le cahier (S6, Élise), juste dans le cahier ( $\mathrm{S} 6$, Tessa). Ces situations d'apprentissage dites authentiques sont surtout des situations authentiquement scolaires qui maintiennent une forme scolaire de relations d'apprentissage (Vincent, Lahire et Thin, 1994). Ces situations demeurent dans l'ici et le maintenant de la réalisation de chaque module. Les pratiques scolaires de l'écrit à l'œuvre dans ces situations demeurent éloignées des situations de la vie courante où le savoir et l'écrit sont dans l'action, notamment chez les adultes des milieux populaires (Bélisle, 2007; Dahl, 2018). Les situations d'apprentissage et les textes qu'elles contiennent sont dans le module et y demeurent. 
Du côté des politiques, la concurrence entre les politiques d'éducation et de formation des adultes et les politiques de réussite éducative qui fait primer la diplomation des adultes sans diplôme du secondaire, notamment celle des jeunes adultes, masque les nombreux buts d'ordre éducatif pourtant présents dans les encadrements normatifs de la formation générale des adultes (Doray et Bélanger, 2014) et ses programmes de formation. Ces politiques et encadrements contribuent, contrairement à leurs objectifs qui s'inscrivent dans le temps long (amener les adultes à tirer des apprentissages de leurs expériences, favoriser leur insertion durable en emploi, ainsi que leur participation sociale, économique et citoyenne, etc.), à maintenir la réalisation des modules dans le moment présent. Les apprenantes comprennent que l'examen, c'est ça qui compte. Le fait que l'accumulation d'unités venant avec la réussite des examens des cours de français, d'anglais et de mathématique soit une exigence de sanction pour la diplomation crée le contexte contribuant au maintien des pratiques de l'écrit afférentes à la réalisation des modules dans le moment présent. Certes, la vision utilitaire de la formation, attachée à ces pratiques de l'écrit, occulte son horizon temporel, futur comme passé, au-delà de son utilité pour le but qui prime dans le moment présent : obtenir le diplôme d'études secondaires ou les préalables à la formation professionnelle ou technique. Mais cette vision utilitaire n'est en bonne partie que la réponse immédiate des jeunes mères au «défi majeur et urgent» (Gouvernement du Québec, 2002, p. 7) que la «norme sociale de référence » (p. 9) en matière de diplomation fait peser sur elles. Comme si l'atteinte de la norme, à elle seule, leur garantissait un avenir meilleur.

\section{Conclusion}

L'étude de cas ethnographique d'où sont tirées les données analysées pour la présente étude montre les pratiques scolaires de l'écrit et les temporalités qui les accompagnent dans l'enseignement modulaire individualisé des cours de français, d'anglais et de mathématique au moment du retour en formation de jeunes mères de Ma place au soleil. Les résultats montrent la centralité de l'évaluation dans ces pratiques et leur maintien dans le temps présent de la formation. Ces résultats sont transférables (Mukamurera, Lacourse et Couturier, 2006) aux contextes de formation similaires à celui enquêté.

Au regard de plusieurs programmes d'études de formation de base commune et de ceux de formation de base diversifiée nouvellement mis en œuvre en blocs d'heures morcelés $(25,50,75$, 
100 heures) ainsi que du maintien de l'organisation scolaire des entrées continues et sorties variables, selon la formule bien connue en formation générale des adultes, il est complexe de remplacer l'enseignement modulaire individualisé et les pratiques scolaires de l'écrit qui l'accompagnent. Non seulement les résultats de la présente étude et des résultats de recherches recensées montrent l'adhésion des jeunes adultes à cette formule, mais celle-ci permet aussi à l'institution de répondre à l'expression de la demande de formation lorsqu'elle se manifeste. Pour élargir l'horizon temporel de la participation à la formation, peut-être vaut-il mieux miser sur la création ou le renforcement des contextes qui font se chevaucher plusieurs volets des services éducatifs de la formation générale des adultes. Il s'agirait de faire cohabiter des services ou des dispositifs qui rendent possibles des pratiques de l'écrit et des temporalités diverses dès le retour en formation des adultes non diplômés, jeunes ou moins jeunes. De tels contextes sont susceptibles de les aider à atteindre leurs objectifs à court terme de diplomation ou d'accès à la formation professionnelle tout en inscrivant leur participation dans leurs expériences passées et dans les perspectives d'avenir.

\section{ENGLISH TITLE-Academic literacy practices and temporalities of modular instruction use in the classroom}

SUMMARY-This article is based on an ethnographic case study conducted with a group of young mothers returning to school in general adult education into the Ma place au soleil program. The goal of this study was to analyze academic literacy practices and temporalities attached to them within individual modular instruction of French, English and mathematics classes. This pedagogical formula is known to be central for these classes of adult general education. The results show the centrality of assessment in these literacy practices and their maintenance in the present time of the training. Exam training influences the maintenance of the literacy practices in present time without showing the relevance of many goals, other than those related to the ones that are state-sanctioned. These results cannot be generalized, but they are transferable to similar contexts to the one studied.

KEYWORDS-literacy practices, temporalities, young mothers, modular instruction, ethnography. 


\section{TíTULO-Prácticas escolares de la escritura y temporalidades en el trabajo por módulos}

RESUMEN-Este artículo presenta un estudio de casos etnográfico desarrollado con un grupo de madres jóvenes de vuelta a los estudios en un programa de formación general de adultos, en el marco de la medida Ma place au soleil. El objetivo del estudio consistió en analizar las prácticas escolares de la escritura y las temporalidades que las acompañan en el trabajo por módulos de los cursos de francés, de inglés y de matemáticas seguidos por estas mujeres. La enseñanza por módulos individualizada es una fórmula reconocida como central en estos cursos de formación general de adultos. Los resultados muestran la centralidad de la evaluación en estas prácticas de la escritura y su mantenimiento en las temporalidades del presente de la formación. El entrenamiento para el examen influencia el mantenimiento de las prácticas de escritura en el tiempo presente, sin dejar ver la pertinencia de otros numerosos objetivos, aparte de aquellos relacionados con la evaluación reglamentada por el Estado. Estos resultados, aunque no son generalizables, son transferibles a los contextos similares al de nuestro estudio.

Palabras Clave-prácticas de escritura, temporalidades, enseñanza por módulos, madres jóvenes, etnografía.

\section{Références}

Baillargeon, R., Gascon, P.-A. et Rivard, A. (1992). L'enseignement individualisé à l'éducation des adultes dans les commissions scolaires. Table des responsables de l'éducation des adultes des commissions scolaires.

Barton, D. et Hamilton, M. (1998). Local literacies. Reading and writing in one community. Routledge.

Bélisle, R. (2003). Pluralité du rapport à l'écrit d'acteurs æuuvrant en milieux communautaires auprès de jeunes adultes peu scolarisés. (Thèse de doctorat). Université de Sherbrooke.

Bélisle, R. (2004). Éducation non-formelle et contribution à l'alphabétisme. Ethnologies, 26(1), 165-183. https://www.erudit.org/fr/revues/ethno/2004-v26-n1-ethno1326/013345ar/

Bélisle, R. (2007). Compétences et pratiques de lecture d'adultes non diplômés : conditions et principes d'un environnement écrit participatif (avec la collaboration de Olivier Dezutter). Ministère de l'Éducation, du Loisir et du Sport.

Bélisle, R. (2012). Introduction. L'écrit pour garder trace et retracer l'apprentissage extrascolaire des adultes. Dans R. Bélisle (dir.), Écrire, lire et apprendre à l'âge adulte (p. 1-20). Presses de l’Université Laval. 
Bélisle, R. et Bourdon, S. (2006). Introduction. Variété, diversité et pluralité des pratiques et de l'apprentissage de l'écrit. Dans R. Bélisle et S. Bourdon (dir.), Pratiques et apprentissage de l'écrit dans les sociétés éducatives (p. 1-27). Presses de l'Université Laval.

Bélisle, R. et Rioux, I. (2016). Recours à l'écrit dans la reconnaissance des acquis au secondaire. Revue canadienne pour l'étude de l'éducation des adultes, 28(1), 1-14. http://cjsae.library.dal.ca/index.php/cjsae/article/view/4791

Bourdon, S. et Bélisle, R. (2005). Temps de rencontre et rencontre de temporalités. L'intervention auprès de jeunes adultes marginalisés comme médiation des temporalités institutionnelles et individuelles. Lien social et politiques, (54), 173-184. https://www.erudit.org/fr/revues/lsp/2005-n54-1sp1053/012869ar/

Bourdon, S. et Bélisle, R. (2011). Programme d'aide pour favoriser le retour en formation de 1624 ans. Le plaisir d'apprendre. J'embarque quand ça me ressemble. Études de cas visant à inspirer de nouveaux environnements éducatifs. Ministère de l'Éducation, du Loisir et du Sport. http://erta.ca/sites/default/files/2017-03/bourdon-belisle_plaisir-apprendre_2011.pdf

Bourdon, S. et Bélisle, R. (2015). Passage à l'âge adulte et précarités dans un monde incertain. Dans S. Bourdon et R. Bélisle (dir.), Les précarités dans le passage à l'âge adulte au Québec (p. 9-26). Les Presses de 1'Université Laval.

Boutinet, J.- P. (2004). Vers une société des agendas. Une mutation des temporalités. Presses universitaires de France.

Conseil du statut de la femme (2004). Avis. Étudiante et mère : un double défi-Les conditions de vie et les besoins des mères étudiantes. Gouvernement du Québec. https://www.csf.gouv.qc.ca/wp-content/uploads/etudiante-et-mere-un-double-defi-lesconditions-de-vie-et-les-besoins-des-meres-etudiantes.pdf

Dahl, A. (2018).Alph@-lab : laboratoire de partage des savoirs. L’Harmattan.

Desmarais, D. (2003). L'alphabétisation en question. Les Éditions Québecor.

Desmarais, D. et Lamoureux, È. (2012). L'apprentissage expérientiel des jeunes adultes en processus de réinsertion sociale. Dans R. Bélisle (dir.), Écrire, lire et apprendre à l'âge adulte (p. 91-110). Presses de 1'Université Laval. 
Doray, P. et Bélanger, P. (2014). Retirer à Pierrette pour donner à Alexandre! Le développement de la formation générale des adultes au Québec. Revue des sciences de l'éducation, 40(2), 215-251. https://www.erudit.org/fr/revues/rse/2014-v40-n2-rse01681/1028420ar/

D’Ortun, F. (2009). La persévérance jusqu'au diplôme du secondaire des 16-25 ans inscrits en zone urbaine et rurale dans les centres de formation aux adultes. Dans N. Rousseau (dir.), Enjeux et défis associés à la qualification: la quête d'un premier diplôme d'études secondaires (p. 77-100). Presses de l’Université du Québec.

Einstein, A. (1905/1923). On the electrodynamics of moving bodies (traduit par W. Perret et G. B. Jeffrey). Dans The principles of relativity publié en 1923 par Methuen and Company Ltd. of London, en allemand Das Relativatsprinzip, (4édition). (D'abord publié en 1922 par Tuebner). http://www.fourmilab.ch/etexts/einstein/specrel/www/

Éliade, M. (1966). Aspects du mythe. Gallimard.

Elias, N. (1996). Du temps (traduit par M. Hulin). Librairie Arthème Fayard.

Goldschmid, B. et Goldschmid, M. L. (1973). Modular instruction in Higher education: A review. Higher Education, 2(1), 15-32.

Goody, J. (1979). La raison graphique. La domestication de la pensée sauvage (traduit par J. Basin et A. Bensa). Les Éditions de Minuit.

Gouvernement du Québec (2002). Politique gouvernementale d'éducation des adultes et de formation continue, apprendre tout au long de la vie. http://www.mess.gouv.qc.ca/publications/pdf/SR_politique_gouv_education_adultes.pdf

Hartog, F. (2012). Régimes d'historicité. Présentisme et expériences du temps. Éditions du Seuil. Ivanic, R., Edwards, R., Barton, R., Martin-Jones, M., Fowler, Z., Hugues, B. et Mannion, G. (2009). Improving learning in college: Rethinking literacies across the curriculum. Routledge.

Legendre, R. (2005). Dictionnaire actuel de l'éducation (3 $3^{\mathrm{e}}$ édition). Guérin éditeur.

Mercier, J.-P. (2015). Pratiques de l'écrit de jeunes mères de retour en formation dans Ma place au soleil. (Thèse de doctorat). Université de Sherbrooke.

Mercier, J.-P. et Longo, M. (2017). Temps de l'écrit et écrit du temps. Imbrication des temps chez des jeunes mères de retour en formation. Recherches féministes, 30(2), 157-174. https://www.erudit.org/fr/revues/rf/2017-v30-n2-rf03510/1043927ar/ 
Merriam, S. B. (1998). Qualitative research and case study applications in education ( $2^{\mathrm{e}}$ édition). Jossey-Bass.

Ministère de l'Éducation du Loisir et du Sport (2007). Programme de la formation de base commune. Document de présentation. Secteur de la formation professionnelle et technique et de la formation continue. Gouvernement du Québec. http://www.education.gouv.qc.ca/fileadmin/site_web/documents/dpse/educ_adulte_action_co $\mathrm{mm} / \mathrm{FBC} \_$Document-de-presentation_fr.pdf

Mukamurera, J., Lacourse, F. et Couturier, Y. (2006). Des avancées en analyse qualitative : pour une transparence et une systématisation des pratiques. Recherches qualitatives, 26(1), 110138. http://www.recherche-qualitative.qc.ca/revue/les-collections/edition-reguliere/

Ouellet, C., Dubeau, A., Dubé, F. et Voyer, B. (2017). Approches et pratiques permettant de diminuer les difficultés en lecture/écriture en français ou en anglais chez les 15-19 ans à la formation générale des adultes et à la formation professionnelle (Rapport no 2015-AP187787). Fonds de recherche du Québec -

Société et culture. http://www.frqsc.gouv.qc.ca/documents/11326/448958/AP_20142015_rapport_OuelletC.pdf/ad31873b-0cc4-4ad9-a288-44cd803c6d1d

Papen, U. (2005). Adult literacy as social practice: More than skills. Routledge.

Potvin, M. et Leclercq, J.-B. (2014). Facteurs affectant la trajectoire scolaire des jeunes de 16-24 ans issus de l'immigration en formation générale des adultes. Revue des sciences de l'éducation, $\quad 40(2), \quad 309-349 . \quad$ https://www.erudit.org/fr/revues/rse/2014-v40-n2rse01681/1028423ar/

Rioux, I. et Bélisle, R. (2012). Présence des autres dans l'écriture de soi d'adultes non diplômés en contexte de reconnaissance des acquis. Dans R. Bélisle (dir.), Écrire, lire et apprendre à l'âge adulte (p. 151-167). Presses de l'Université Laval.

Rousseau, N., Théberge, N., Bergevin, S., Tétreault, K., Samson, G., Dumont, M. et MyreBisaillon, J. (2010). L'éducation des adultes chez les 16-18 ans: la volonté de réussir l'école... et la vie! Éducation et francophonie, 38(1), 154-177. https://www.erudit.org/fr/revues/ef/2010-v38-n1-ef3866/039985ar/ 
Savoie-Zajc, L. (2004). La recherche qualitative/interprétative en éducation. Dans Karsenti, T. et Savoie-Zajc, L. (dir.), La recherche en éducation : étapes et approches (p. 123-150). Éditions du CRP.

Thériault, V. (2015). Literacy mediation as a form of powerful literacies in community-based organisations working with young people in a situation of precarity. Ethnography and Education, 11(2), 158-173. http://dx.doi.org/10.1080/17457823.2015.1101384

Torrance, H., Colley, H., Garratt, D., Jarvis, J., Piper, H. et Ecclestone, K. (2005). The impact of different modes of assessment on achievement and progress in the learning and skills sector. Learning and Skills Development Agency. http://www.itslifejimbutnotasweknowit.org.uk/files/AssessmentModesImpact.pdf

Vincent, G., Lahire, B. et Thin, D. (1994). Sur l'histoire et la théorie de la forme scolaire. Dans G. Vincent (dir.), L'éducation prisonnière de la forme scolaire ? Scolarisation et socialisation dans les sociétés industrielles (p. 11-48). Presses universitaires de Lyon.

Voyer, B., Potvin, M. et Bourdon, S. (2014). Les transformations et défis actuels de la formation générale des adultes. Revue des sciences de l'éducation, 40(2), 191-213. https://www.erudit.org/fr/revues/rse/2014-v40-n2-rse01681/1028419ar/

Vygotski, L. (1997). Pensée et langage (traduit par F. Sève). Éditions La Dispute/SNÉDIT.

\section{Correspondance}

mercier.jean-pierre@uqam.ca

\section{Contribution de l'auteur}

Jean-Pierre Mercier : $100 \%$

Ce texte a été révisé par : Mélissa Singcaster

Texte reçu le : 3 septembre 2018

Version finale reçue le : $1^{\text {er }}$ octobre 2019

Accepté le : 23 décembre 2019 